\title{
Spectral Efficiency Optimization with Distributed Beamforming in UWB Based Implant Body Area Networks
}

\author{
Jie Ding*, Eryk Dutkiewicz* ${ }^{*}$ Xiaojing Huang ${ }^{\dagger}$ \\ *Department of Engineering, Macquarie University, Australia \\ \{jie.ding1, eryk.dutkiewicz\}@mq.edu.au \\ $\dagger$ CSIRO Computational Informatics, Australia \\ Xiaojing.Huang@csiro.au
}

\begin{abstract}
In this paper, the SE optimization based distributed beamforming problem is investigated for ultra-wideband (UWB) based implant body area networks (IBANs). We consider a relay network of one implant source, several wearable relays, and one body network coordinator under the assumption that the individual relay power is constrained due to Federal Communications Commission (FCC) regulations for UWB signal. Taking into account realistic wireless channels and relay locations, the $\mathrm{SE}$ optimization problem is mathematically formulated and solved by using convex optimization. Simulation results show that the proposed beamforming scheme is superior to other transmission schemes. Moreover, our numerical examples reveal that the relay location has a significant impact on the beamforming performance and the proposed beamforming scheme can be considered as an efficient way to prolong the lifetime of the implanted node.
\end{abstract}

\section{INTRODUCTION}

Wireless body area network (BAN) is an enabling technology for pervasive healthcare by using several sensors on/in the human body [1], [2]. Ultra-wideband (UWB) technology has great potential for applications in BAN, owing to its simple electronics and low power consumption. In UWB based implant BANs (IBANs), the achievable data rate of direct transmission is often unsatisfactory because of the propagation blockage from the body torso as well as the low transmit power. Thus, spectral efficiency (SE), as a performance criterion of the data rate, is one of the most critical concerns for UWB based IBANs. On the other hand, cooperative communication techniques have gained much attention in wireless networks which can achieve cooperative diversity gain that improves link reliability and SE [3].

Considerable cooperative transmission strategies have been conducted in wireless sensor networks (WSNs) regarding the SE [4]-[7]. In [4], single relay selection scheme was proposed to achieve the maximum SE and minimum outage probability. For multiple relay cooperation with perfect channel state information (CSI), distributed beamforming strategies have been developed to maximum SE subject to different power constrains under flat fading channels [5], [6]. In [7], the techniques of [5], [6] has been extended to the frequency selective fading channel scenarios. While aforementioned work mainly focused on the narrowband cases, it is not straightforward to apply for the impulse-based UWB cases due to the special properties of UWB signaling such as analog transmission and dense resolvable multipath. Thus, some extensions of cooperative transmission strategies to UWB systems have been known in literature [8]-[11]. However, the problem of distributed beamforming regarding SE for UWB based BANs is still an open issue.

Unlike conventional networks, BANs have many unique properties, e.g. distinct channel characteristics and very small network size, where the signal strength is mostly affected by the physical location of the nodes in relation to each other as well as the human body. For relay based cooperative BANs, the impact of relay location and channel path loss between nodes must be taken into account therefore. In particular, both "in-body to on-body" and "on-body to on-body" channels are involved in cooperative IBANs, which make the relay location have a critical impact on the system performance. Thus, it is worth to analyze the performance of distributed beamforming and evaluate the effect of relay location in UWB based BANs.

In this paper, the SE optimization based distributed beamforming problem is developed for UWB based IBANs. Specifically, an IBAN consists of one implanted transmitter (Source), several parallel wearable relays, and one body network coordinator (Destination) is considered. At destination and each relay, Rake receiver and match filter are employed to capture the multipath gain, respectively. Benefit from this configuration, the proposed network beamforming scheme is equivalent to solve a distributed power allocation problem, where each relay properly adjust its own power such that the system SE can be maximized. With the assumption that individual relay power is constrained due to Federal Communications Commission (FCC) regulations for UWB signal, the network beamforming problem can be reduced to a quasiconvex optimization problem, which can be solved by using convex optimization. Simulations results show that the proposed beamforming scheme outperforms other transmission schemes. Moreover, the relay location plays an important role on the beamforming 
performance and our numerical examples reveal that the optimal relay location varies with the implanted transmit power. Furthermore, given a targeted SE, the implanted power consumption is less as the larger the number of relays and the rake fingers at the coordinator are employed, which indicates the proposed beamforming scheme can be considered as an efficient way to prolong the lifetime of implanted node.

The remainder of this paper is organized as follows. In Section II, the system and channel models are described. In Section III, the SE maximization for distributed beamforming is detailed. Simulation results are presented in Section IV and the paper is concluded in Section V.

\section{System ANd Channel Models}

\section{A. System Scenarios}

Since two-hop cooperative communication is specified as an optional scheme in the IEEE 802.15.6 standard [1], the twohop cooperative communication is considered in this paper. As shown in Fig. 1, a cooperative model for IBANs is proposed, where the transmitter node $S$ (Source) is implanted in the human chest, and $m$ wearable relay nodes $\left(R_{i}\right.$, for $\left.\mathrm{i}=1, \ldots, m\right)$ and a body network coordinator $D$ (Destination) are assumed to be on the same side of body surface. We denote the distance from $S$ to $D$ and penetration depth from $S$ to the body surface by $d_{S D}$ and $d_{r}$, respectively. On the body surface, we construct a xy-plane to present coordinates of $m$ relays, where $P$ is the projection of $S$ on the body surface and set to be the origin point, and the $\mathrm{x}$-axis is along $P$ to $D$. In this paper, a special parallel relay topology is considered, where $m$ relays have the same $x$-coordinate $x_{0}[\mathrm{~mm}]\left(x_{0} \geq 0\right)$ and are evenly distributed at $\mathrm{y}$-axis. With a given $y$-coordinate $y_{i}[\mathrm{~mm}]$ for relay $i$, we have

$$
d_{S R_{i}}=\sqrt{x_{0}^{2}+y_{i}^{2}+d_{r}^{2}},
$$

and

$$
d_{R_{i} D}=\sqrt{\left(x_{0}-d_{S D} \sin \theta\right)^{2}+y_{i}^{2}},
$$

where $d_{S R_{i}}$ and $d_{R_{i} D}$ are the distances from $S$ to relay $i$ and relay $i$ to $D$, respectively. $\theta=\arccos \left(d_{r} / d_{s d}\right)$. It is worth to note that we herein study a two-step amplify-and-forward (AF) protocol. In the first step, $S$ broadcasts its signal to relays, and during the second step, relays forwards its received signal to $D$.

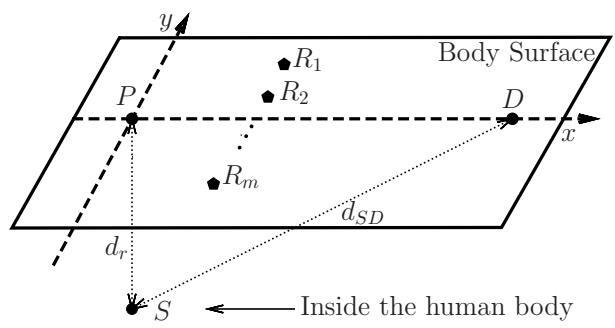

Fig. 1. Proposed cooperative model for IBANs.

\section{B. Channel Models}

For cooperative transmission, two types of channel models need to be considered in UWB based IBANs, namely "alongtorso" channel model from relays to $D$ and "in-body" channel model from $S$ to relays. Table I summarizes the path loss models with corresponding parameters [12], [13].

TABLE I

UWB BASED BAN PATH LOSS MODELS

\begin{tabular}{|c|c|}
\hline "along-torso" channel & $P L_{0}^{d B}(d)=P_{0}+10 n_{0} \log _{10}\left(\frac{d}{d_{r 0}}\right)$ \\
\hline$P_{0}[\mathrm{~dB}]$ & 44.6 \\
\hline$n_{0}$ & 3.1 \\
\hline$d_{r 0}[m]$ & 0.1 \\
\hline \hline "in-body" channel & $P L_{1}^{d B}(d)=P_{1}+a\left(d / d_{r 1}\right)^{n_{1}}+H$ \\
\hline$P_{1}[\mathrm{~dB}]$ & 10 \\
\hline$a$ & 0.987 \\
\hline$n_{1}$ & 0.85 \\
\hline$d_{r 1}[m]$ & 0.001 \\
\hline$H$ & $\mathcal{N}(0,61.47)$ \\
\hline
\end{tabular}

${ }^{1} P_{i}$ is the path loss at the reference distance, for $\mathrm{i}=0,1$.

${ }^{2} d_{r i}$ is the reference distance, for $\mathrm{i}=0,1$.

${ }^{3} n_{i}$ is the path loss exponent, for $\mathrm{i}=0,1$.

${ }^{4} a$ is a fitting constant.

${ }^{5} H$ is a Gaussian distributed random variable with zero mean and standard deviation 7.84 .

From the path loss models defined in the log scale, we can obtain the path losses in the linear scale from $S$ to $R_{i}$ and $R_{i}$ to $D$ as,

$$
P L_{S R_{i}}\left(d_{S R_{i}}\right)=10^{P L_{1}^{d B}\left(d_{S R_{i}}\right) / 10}, \quad i=1, \ldots, m
$$

and

$$
P L_{R_{i} D}\left(d_{R_{i} D}\right)=10^{P L_{0}^{d B}\left(d_{R_{i} D}\right) / 10}, \quad i=1, \ldots, m
$$

respectively.

For all the links considered, the energy-normalized channel impulse response (CIR) can be written as

$$
h_{k}(t)=\sum_{l=0}^{L_{k}-1} \alpha_{l, k} \delta\left(t-\tau_{l, k}\right),
$$

where $k \in\left\{S R_{i}, R_{i} D\right\}$ denotes the links from $S$ to $R_{i}$ and $R_{i}$ to $D$ respectively. $L_{k}$ is the number of multipaths, $\tau_{l, k}$ is the delay of the $l$ th path, and $\alpha_{l, k}$ is the gain of the $l$ th path. Since real signals is employed in UWB systems, we are only interested in the real part of each path gain. The further detail on the delay profile for "along-torso" and "in-body" links can be found in [12] and [13], respectively.

\section{Signal Model}

For analytical simplicity, we present the IR-UWB signal model with pulse-amplitude modulation (PAM). The random time hopping (TH) or direct spread (DS) codes are omitted in peer to peer communications.

At $S$, every transmitted PAM symbol duration $T_{s}$ consists of $N_{f}$ consecutive frames each with duration $T_{f}$, where $T_{s}=$ $N_{f} T_{f}$. The symbol information is conveyed by a ultrashort 
pulse waveform in each frame. Thus the transmitted symbol waveform can be expressed as

$$
s(t)=b \sqrt{P_{s}} \sum_{j=0}^{N_{f}-1} \omega\left(t-j T_{f}\right), b= \pm 1
$$

where $b$ is the transmitted symbol and $P_{s}$ is the implanted transmit power and the value of $P_{s}$ depends on the battery power limit of the implanted node. $\omega(t)$ denotes the ultrashort pulse waveform with $T_{w}$ duration, which has the unit energy $\int_{t=0}^{T_{f}} \omega^{2}(t)=1$.

For all the links, it is assumed that channel $h_{k}(t)$ remains invariant over a symbol duration, but it changes from symbol to symbol. Thus, the received signal at relay $i$ is given by

$$
\begin{aligned}
r_{S R_{i}}(t)= & b \sqrt{\frac{P_{s}}{P L_{S R_{i}}\left(d_{S R_{i}}\right)} \sum_{j=0}^{N_{f}-1} g_{S R_{i}}\left(t-j T_{f}\right)} \\
& +n_{S R_{i}}(t),
\end{aligned}
$$

where

$$
g_{S R_{i}}(t)=\omega(t) * h_{S R_{i}}(t)=\sum_{l=0}^{L_{S R_{i}}-1} \alpha_{l, S R_{i}} \omega\left(t-\tau_{l, S R_{i}}\right)
$$

and $n_{S R_{i}}$ is the additive white Gaussian noise (AWGN) with zero mean and variance $\sigma^{2}$.* represents convolution. $T_{f}$ is set to be large enough to avoid the inter-symbol interference (ISI).

At each relay, the received pulse waveform matched filter is employed. During each frame duration, the output of the matched filter at relay $i$ is given by

$$
\begin{aligned}
\bar{r}_{S R_{i}}(j) & =\int_{j T_{f}}^{(j+1) T_{f}} r_{S R_{i}}(t) g_{S R_{i}}\left(t-j T_{f}\right) d t \\
& =b \sqrt{\frac{P_{s}}{P L_{S R_{i}}\left(d_{S R_{i}}\right)}} \xi_{T_{f}}\left(h_{S R_{i}}\right)+\bar{n}_{S R_{i}}(j),
\end{aligned}
$$

where $j \in\left[0, N_{f}-1\right] . \xi_{T_{f}}\left(h_{S R_{i}}\right)$ is the captured multipath gain during $T_{f}$ at relay $i$ and $\bar{n}_{S R_{i}}(j)$ is still a white Gaussan noise with zero mean and variance $\xi_{T_{f}}\left(h_{S R_{i}}\right) \sigma^{2}$.

After summing up all the outputs over $N_{f}$ frames, the decision statistic $b$ at relay $i$ can be written as

$$
b_{R_{i}}=b N_{f} \sqrt{\frac{P_{s}}{P L_{S R_{i}}\left(d_{S R_{i}}\right)}} \xi_{T_{f}}\left(h_{S R_{i}}\right)+\underbrace{\sum_{j=0}^{N_{f}-1} \bar{n}_{S R_{i}}(j)}_{\hat{n}_{S R_{i}}} .
$$

With $\left\{b_{R_{i}}\right\}_{i=1}^{m}$, distributed space-time block coding (STBC) is applied at relays to achieve spatial diversity gain [14]. Let B be the STBC matrix with dimension $m \times N_{f}^{\prime}$, where $N_{f}^{\prime}$ is the block length. Examples of the STBC matrix are shown in Table II. In this paper, we assume that $N_{f}=Q N_{f}^{\prime}$ and $Q$ is an integer. Thus, $N_{f}$ frames are divided into $Q$ frame blocks and the transmitted signal vector from relays in the second hop can be modeled as

$$
\mathbf{t}_{R}(t)=\sqrt{P_{\max }} \sum_{q=1}^{Q} \hat{\mathbf{b}}_{R} \hat{\mathbf{K}}_{R} \hat{\mathbf{W}}_{R} \mathbf{B} \boldsymbol{\omega}\left(t-N_{f} T_{f}, q\right),
$$

where $\mathbf{t}_{R}(t)=\left[t_{R_{1}}(t), t_{R_{2}}(t), \ldots, t_{R_{m}}(t)\right]^{T}$ and the element $t_{R_{i}}(t)$ represents the transmitted signal at relay i. $P_{\max }$ is the maximum transmit power at each relay, which is constrained by FCC regulations. $\hat{\mathbf{b}}_{R} \triangleq$ $\operatorname{diag}\left\{b_{R_{1}}, \ldots, b_{R_{m}}\right\} . \hat{\mathbf{K}}_{R} \triangleq \operatorname{diag}\left\{\left|b_{R_{1}}\right|^{-1}, \ldots,\left|b_{R_{m}}\right|^{-1}\right\}$ and $\left|b_{R_{i}}\right|=\sqrt{\frac{N_{f}^{2} P_{s} \xi_{T_{f}}^{2}\left(h_{S R_{i}}\right)}{P L_{S R_{i}}\left(d_{S R_{i}}\right)}+N_{f} \xi_{T_{f}}\left(h_{S R_{i}}\right) \sigma^{2}} \cdot \hat{\mathbf{W}}_{R} \triangleq$ $\operatorname{diag}\left\{w_{1}, \ldots, w_{m}\right\}$ is the diagonal matrix of the relay power allocation weights and $0 \leq w_{i} \leq 1$. Clearly, the transmit power of relay $i$ is $w_{i}^{2} P_{\text {max }}$. We define $\boldsymbol{\omega}(t, q) \triangleq[\omega(t-$ $\left.N_{f}^{\prime}(q-1) T_{f}\right), \omega\left(t-N_{f}^{\prime}(q-1) T_{f}-T_{f}\right), \ldots, \omega\left(t-N_{f}^{\prime}(q-\right.$ 1) $\left.\left.T_{f}-\left(N_{f}^{\prime}-1\right) T_{f}\right)\right]^{T}$ is the waveform vector with length $N_{f}^{\prime}$.

TABLE II

EXAMPLES OF B FOR RELAYS

\begin{tabular}{|c|c|c|}
\hline$m$ & $N_{f}^{\prime}$ & B \\
\hline \hline 2 & 2 & $\left(\begin{array}{cc}1 & -1 \\
1 & 1\end{array}\right)$ \\
\hline 3 & 4 & $\left(\begin{array}{cccc|}1 & -1 & -1 & -1 \\
1 & 1 & 1 & -1 \\
1 & -1 & 1 & 1\end{array}\right)$ \\
\hline \multirow{3}{*}{4} & \multirow{2}{*}{4} & $\left(\begin{array}{cccc}1 & -1 & -1 & -1 \\
1 & 1 & 1 & -1 \\
1 & -1 & 1 & 1 \\
1 & 1 & -1 & 1\end{array}\right)$ \\
\hline
\end{tabular}

After passing through channels from relays to $D$, the received signal at $D$ can be represented by

$$
\begin{aligned}
r_{D}(t)= & \sqrt{P_{\max }} \sum_{q=1}^{Q} \sum_{i=1}^{m} \frac{\mathbf{e}_{i}^{T} \hat{\mathbf{b}}_{R} \hat{\mathbf{K}}_{R} \hat{\mathbf{W}}_{R} \mathbf{B g}_{R_{i} D}\left(t-N_{f} T_{f}, q\right)}{\sqrt{P L_{R_{i} D}\left(d_{R_{i} D}\right)}} \\
& +n_{D}(t),
\end{aligned}
$$

where $\mathbf{e}_{i}$ is the $i$ th column of the identity matrix. $\mathbf{g}_{R_{i} D}(t, q) \triangleq\left[g_{R_{i} D}\left(t-N_{f}^{\prime}(q-1) T_{f}\right), g_{R_{i} D}\left(t-N_{f}^{\prime}(q-\right.\right.$ 1) $\left.\left.T_{f}-T_{f}\right), \ldots, g_{R_{i} D}\left(t-N_{f}^{\prime}(q-1) T_{f}-\left(N_{f}^{\prime}-1\right) T_{f}\right)\right]^{T}$ and $g_{R_{i} D}(t)=\omega(t) * h_{R_{i} D}(t) . n_{D}(t)$ is the AWGN with zero mean and variance $\sigma^{2}$.

At $D$, a Rake receiver with $L_{r}$ fingers is employed and the delayed reference waveforms $\left\{\omega\left(t-\tau\left(l_{r}\right)\right)\right\}_{l_{r}=0}^{L_{r}-1}$ are used. After combining the Rake outputs over $N_{f}^{\prime}$ frames in each frame block, the output per frame block can be written as

$$
\begin{aligned}
& z_{D}=\sqrt{P_{\max }} \sum_{l_{r}=0}^{L_{r}-1} \boldsymbol{\beta}_{R D}^{T}\left(l_{r}\right) \mathbf{B B}^{T} \hat{\mathbf{K}}_{R} \hat{\mathbf{W}}_{R} \hat{\mathbf{P L}}_{R D} \mathbf{b}_{R}+\bar{n}_{D}(t) \\
& =N_{f}^{\prime} \sqrt{P_{\max }} \boldsymbol{\xi}_{R D}^{T} \hat{\mathbf{K}}_{R} \hat{\mathbf{W}}_{R} \hat{\mathbf{P L}} \hat{\mathbf{L}}_{R D} \mathbf{b}_{R}+\bar{n}_{D}
\end{aligned}
$$

where $l_{r} \in\left[0, L_{r}-1\right] . \mathbf{b}_{R}=\left[b_{R_{1}}, \ldots, b_{R_{m}}\right]^{T}$. $\boldsymbol{\beta}_{R D}\left(l_{r}\right) \triangleq \quad\left[\beta_{R_{1} D}^{2}\left(l_{r}\right), \ldots, \beta_{R_{m} D}^{2}\left(l_{r}\right)\right]^{T} \quad$ and 
$\beta_{R_{i} D}\left(l_{r}\right)=\int_{0}^{T_{f}} g_{R_{i} D} \omega\left(t-\tau\left(l_{r}\right)\right) d t . \quad \hat{\mathbf{P L}}_{R D} \triangleq$ $\operatorname{diag}\left\{P L_{R_{1} D}^{-\frac{1}{2}}\left(d_{R_{1} D}\right), \ldots, P L_{R_{m} D}^{-\frac{1}{2}}\left(d_{R_{m} D}\right)\right\} . \quad \boldsymbol{\xi}_{R D} \triangleq$ $\left[\xi_{R_{1} D}, \ldots, \xi_{R_{m} D}\right]^{T}$ and $\xi_{R_{i} D}=\sum_{l_{r}=0}^{L_{r}-1} \beta_{R_{i} D}^{2}\left(l_{r}\right)$. Notice that $\xi_{R_{i} D}$ represents the captured multipath gain from link $R_{i}$ to $D . \bar{n}_{D}$ is the AWGN with zero mean and variance $N_{f}^{\prime} \sum_{i=1}^{m} \xi_{R_{i} D} \sigma^{2}$.

Summing up all outputs over $Q$ frame blocks and substituting (5) into (8), the decision variable for $b$ can be given by

$$
\begin{aligned}
\hat{z} & =N_{f} \sqrt{P_{\max }} \boldsymbol{\xi}_{R D}^{T} \hat{\mathbf{K}}_{R} \hat{\mathbf{W}}_{R} \hat{\mathbf{P L}} \hat{L}_{R D} \mathbf{b}_{R}+\hat{n}_{D} \\
& =b N_{f}^{2} \sqrt{P_{\max } P_{s}} \boldsymbol{\xi}_{R D}^{T} \hat{\mathbf{K}}_{R} \hat{\mathbf{W}}_{R} \hat{\mathbf{P L}} \hat{\mathbf{L}}_{R D} \hat{\mathbf{P L}}{ }_{S R} \boldsymbol{\xi}_{S R} \\
& +N_{f} \sqrt{P_{\max }} \boldsymbol{\xi}_{R D}^{T} \hat{\mathbf{K}}_{R} \hat{\mathbf{W}}_{R} \hat{\mathbf{P L}}{ }_{R D} \hat{\mathbf{n}}_{S R}+\hat{n}_{D},
\end{aligned}
$$

where $\hat{\mathbf{P L}}_{S R} \triangleq \operatorname{diag}\left\{P L_{S R_{1}}^{-\frac{1}{2}}\left(d_{S R_{1}}\right), \ldots, P L_{S R_{m}}^{-\frac{1}{2}}\left(d_{S R_{m}}\right)\right\}$. $\boldsymbol{\xi}_{S R} \triangleq\left[\xi_{T_{f}}\left(h_{S R_{1}}\right), \ldots, \xi_{T_{f}}\left(h_{S R_{m}}\right)\right]^{T} . \quad \hat{\mathbf{n}}_{S R} \triangleq$ $\left[\hat{n}_{S R_{1}}, \ldots, \hat{n}_{S R_{m}}\right]^{T} . \hat{n}_{D}$ is the AWGN with zero mean and variance $N_{f} \sum_{i=1}^{m} \xi_{R_{i} D} \sigma^{2}$.

In (9), we identify three components

$$
\begin{gathered}
\hat{z}_{s}=b N_{f}^{2} \sqrt{P_{\max } P_{s}} \boldsymbol{\xi}_{R D}^{T} \hat{\mathbf{K}}_{R} \hat{\mathbf{W}}_{R} \hat{\mathbf{P L}} \hat{\mathbf{L}}_{R D} \hat{\mathbf{P L}}{ }_{S R} \boldsymbol{\xi}_{S R}, \\
\hat{z}_{n_{S R}}=N_{f} \sqrt{P_{\max }} \boldsymbol{\xi}_{R D}^{T} \hat{\mathbf{K}}_{R} \hat{\mathbf{W}}_{R} \hat{\mathbf{P L}}_{R D} \hat{\mathbf{n}}_{S R}
\end{gathered}
$$

and

$$
\hat{z}_{n_{R D}}=\hat{n}_{D},
$$

as the useful signal, noise from the first hop, and noise from the second hop, respectively.

Obviously, the SE optimization based beamforming design is to find the optimal $\mathbf{w}_{R}=\left[w_{1}, \ldots, w_{m}\right]^{T}$ to maximize the receive SE, which is equivalent to the optimal relay power allocation. In next section, the optimal relay power allocation in (9) is investigated.

\section{Optimal Relay Power Allocation}

In this section, we analyze the optimal relay power allocation at relays. Since SE is a monotonically increasing function of the receive signal to noise ratio (SNR), the receive SNR is optimized accordingly.

Based on (9)-(12), the receive $\mathrm{SNR} \mathrm{SNR}_{D}$ can be expressed as

$$
\operatorname{SNR}_{D}\left(\mathbf{w}_{R}\right)=\frac{\mathrm{E}\left\{\left|\hat{z}_{s}\right|^{2}\right\}}{\mathrm{E}\left\{\left|\hat{z}_{n_{S R}}\right|^{2}\right\}+\mathrm{E}\left\{\left|\hat{z}_{n_{R D}}\right|^{2}\right\}} .
$$

Using (10), we have that

$$
\mathrm{E}\left\{\left|\hat{z}_{s}\right|^{2}\right\}=\mathbf{w}_{R}^{T} \mathbf{O}_{s} \mathbf{w}_{R},
$$

where $\mathbf{O}_{s}=N_{f}^{4} P_{\max } P_{s} \mathbf{V} \mathbf{V}^{T}$ and $\mathbf{V}=$ $\hat{\boldsymbol{\xi}}_{R D} \hat{\mathbf{K}}_{R} \hat{\mathbf{P L}}_{R D} \hat{\mathbf{P L}}_{S R} \boldsymbol{\xi}_{S R} . \hat{\boldsymbol{\xi}}_{R D}=\operatorname{diag}\left\{\xi_{R_{1} D}, \ldots, \xi_{R_{m} D}\right\}$.

Using (11), we have that

$$
\mathrm{E}\left\{\left|\hat{z}_{n_{S R}}\right|^{2}\right\}=\mathbf{w}_{R}^{T} \mathbf{O}_{n} \mathbf{w}_{R}
$$

where $\mathbf{O}_{n}=N_{f}^{3} P_{\max } \sigma^{2} \hat{\boldsymbol{\xi}}_{R D}^{2} \hat{\mathbf{K}}_{R}^{2} \hat{\mathbf{P L}}_{R D}^{2} \hat{\boldsymbol{\xi}}_{S R}$ and $\hat{\boldsymbol{\xi}}_{S R}=$ $\operatorname{diag}\left\{\xi_{T_{f}}\left(h_{S R_{1}}\right), \ldots, \xi_{T_{f}}\left(h_{S R_{m}}\right)\right\}$.
From (12), we obtain that

$$
\mathrm{E}\left\{\left|\hat{z}_{n_{R D}}\right|^{2}\right\}=N_{f} \sum_{i=1}^{m} \xi_{R_{i} D} \sigma^{2} .
$$

Using (13)-(16), the distributed beamforming problem can be written as

$$
\begin{array}{cl}
\underset{\mathbf{w}_{R}}{\operatorname{maximize}} & \frac{\mathbf{w}_{R}^{T} \mathbf{O}_{s} \mathbf{w}_{R}}{\mathbf{w}_{R}^{T} \mathbf{O}_{n} \mathbf{w}_{R}+N_{f} \sum_{i=1}^{m} \xi_{R_{i} D} \sigma^{2}} \\
\text { subject to } & \mathbf{0}_{m} \preceq \mathbf{w}_{R} \preceq \mathbf{I}_{m},
\end{array}
$$

where $\mathbf{0}_{m}$ is $m$-dimensional vector with all zero entries and $\mathbf{I}_{m}$ is $m$-dimensional vector with all one entries. With two $m$-dimensional vectors $\mathbf{a}$ and $\mathbf{c}, \mathbf{a} \preceq \mathbf{c}$ means $a_{i} \leq c_{i}$ for all $i \in[1, m]$.

With an auxiliary variable $\delta$ [15], (17) is equivalent to

$$
\begin{array}{ll}
\underset{\mathbf{w}_{R}, \delta}{\operatorname{maximize}} & \delta \\
\text { subject to } & \frac{\mathbf{w}_{R}^{T} \mathbf{O}_{s} \mathbf{w}_{R}}{\mathbf{w}_{R}^{T} \mathbf{O}_{n} \mathbf{w}_{R}+N_{f} \sum_{i=1}^{m} \xi_{R_{i} D} \sigma^{2}} \geq \delta^{2} \\
& \mathbf{0}_{m} \preceq \mathbf{w}_{R} \preceq \mathbf{I}_{m} .
\end{array}
$$

Introducing that

$$
\begin{gathered}
\mathbf{U}^{T} \mathbf{U} \triangleq\left(\begin{array}{cc}
N_{f} \sum_{i=1}^{m} \xi_{R_{i} D} \sigma^{2} & \mathbf{0}_{m}^{T} \\
\mathbf{0}_{m} & \mathbf{O}_{n}
\end{array}\right), \\
\tilde{\mathbf{w}}_{R} \triangleq\left[1, \mathbf{w}_{R}^{T}\right]^{T},
\end{gathered}
$$

and

$$
\tilde{\mathbf{V}} \triangleq\left[0, \mathbf{V}^{T}\right]^{T}
$$

Then, (18) can be further written as

$$
\begin{array}{cl}
\underset{\tilde{\mathbf{w}}_{R}, \delta}{\operatorname{maximize}} & \delta \\
\text { subject to } & N_{f}^{2} \sqrt{P_{\max } P_{s}} \tilde{\mathbf{w}}_{R}^{T} \tilde{\mathbf{V}} \geq \delta\left\|\mathbf{U} \tilde{\mathbf{w}}_{R}\right\| \\
& \mathbf{0}_{m} \preceq \tilde{\mathbf{w}}_{R} \preceq \mathbf{I}_{m} \\
& \tilde{\mathbf{w}}_{R}^{T} \mathbf{e}_{1}=1 .
\end{array}
$$

Since (22) is a quasiconvex problem, it can be reduced to the following feasibility problem

$$
\begin{array}{ll}
\text { find } & \tilde{\mathbf{w}}_{R} \\
\text { subject to } & N_{f}^{2} \sqrt{P_{\max } P_{s}} \tilde{\mathbf{w}}_{R}^{T} \tilde{\mathbf{V}} \geq \delta\left\|\mathbf{U} \tilde{\mathbf{w}}_{R}\right\| \\
& \mathbf{0}_{m} \preceq \tilde{\mathbf{w}}_{R} \preceq \mathbf{I}_{m} \\
& \tilde{\mathbf{w}}_{R}^{T} \mathbf{e}_{1}=1 .
\end{array}
$$

With (22) and (23), the bisection search method can be used to find the optimal power allocation vector $\mathbf{w}_{R}^{o}$ [15]. Accordingly, the optimal SE can be given by $\frac{1}{2 N_{f}} \log (1+$ $\left.S N R_{D}\left(\mathbf{w}_{R}^{o}\right)\right)[\mathrm{bits} / \mathrm{s} / \mathrm{Hz}]$. 


\section{Simulation}

To assess the SE of the proposed distributed beamforming scheme in UWB based IBANs, numerical results and examples are conducted in this section. In simulations, $N_{f}$ is set to be 4. The noise power spectral density (PSD) is $-174 \mathrm{dBm} / \mathrm{Hz}$ and the system bandwidth is $500 \mathrm{MHz}$. Since the FCC PSD emission limit for UWB signal is $-41.3 \mathrm{dBm} / \mathrm{MHz}, P_{\max }$ is set to be $-14.3 \mathrm{dBm}$. According to the scale of the human body, $d_{S D}$ and $d_{r}$ are set to be $200 \mathrm{~mm}$ and $50 \mathrm{~mm}$, respectively. At relays, we assume that $\xi_{T_{f}}\left(h_{S R_{i}}\right) \approx 1$ for all $i \in[1, m]$ without considering the ISI. At $D$, selective Rake receiver is employed to combine the $L_{r}$ strongest multipath components.

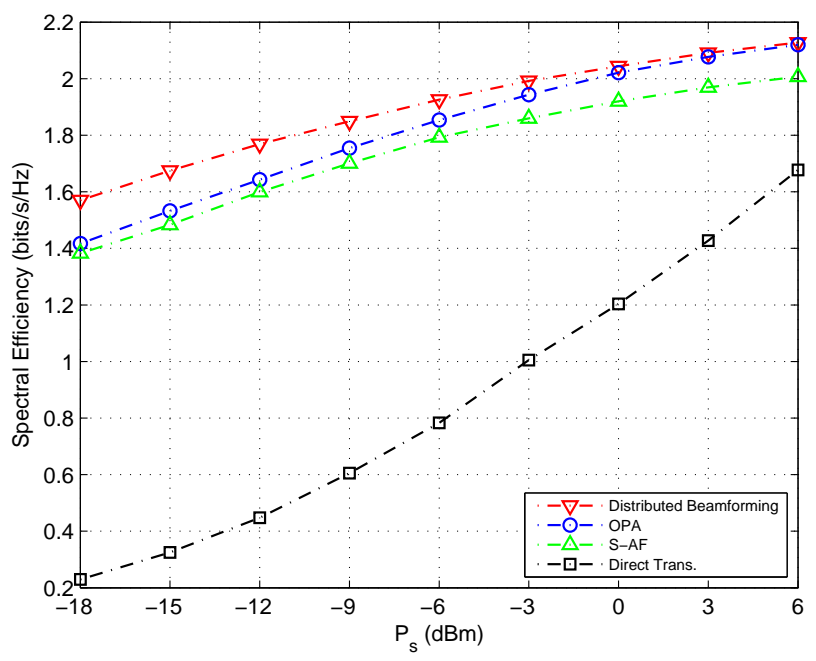

Fig. 2. Average SE versus $P_{s}$ in a 2-relay network.

Fig. 2 shows the average $\mathrm{SE}$ versus $P_{s}$ in a 2-relay UWB based IBANs. For this 2-relay case, we assume that the coordinates of two relays are set to be $\{(90,20)\}$ and $\{(90,-20)\}$, respectively. Performance of the proposed distributed beamforming scheme is compared to those of equal power allocation (OPA) scheme (every relay uses its maximum power), best-relay selection (S-AF) scheme, and direct transmission. We can see that the proposed distributed beamforming scheme outperforms all other schemes. With perfect channel information at relays, it is about $5 \mathrm{~dB}$ better than OPA scheme at low-to-moderate $P_{s}$ regime which needs no channel information about the second hop at relays. Compared to direct transmission, we can see that the proposed distributed beamforming scheme can provide more than $20 \mathrm{~dB}$ performance improvement and this improvement mainly results from the fact that the path loss of the "in-body to on-body" link is much stronger than that of the "on-body to on-body" link. From the perspective of power consumption, the transmit power at $S$ by using distributed beamforming scheme is $20 \mathrm{~dB}$ less compared to that by direct transmission when the same SE is achieved. This evidence indicates that the lifetime of the implanted node can be prolonged considerably in distributed relay networks, which demonstrates the effectiveness of the proposed distributed beamforming scheme applied to UWB based IBANs.

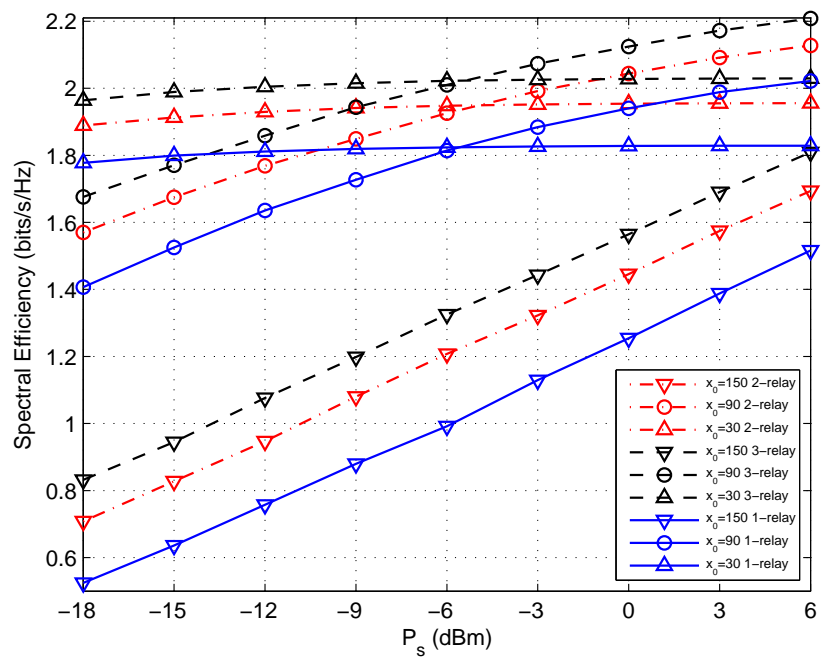

Fig. 3. Average SE versus $P_{s}$ with different number of relays and relay location.

Fig. 3 depicts the average SE versus $P_{s}$ with different number of relays and relay location. Specifically, three cases with $m=1,2$, and 3 are considered, respectively. For the case $m=1$, the coordinate of single relay is set to be $\left\{\left(x_{0}, 0\right)\right\}$. For the case $m=2$, the coordinates of relays are set to be $\left\{\left(x_{0}, 20\right),\left(x_{0},-20\right)\right\}$. For the case $m=3$, the coordinates of relays are set to be $\left\{\left(x_{0}, 20\right),\left(x_{0}, 0\right),\left(x_{0},-20\right)\right\}$. For all the cases, $x_{0}$ is set to be 30,90 , and 150 , respectively, where $x_{0}=30$ represents that relays close to $P, x_{0}=90$ represents that relays located near the middle of $P$ and $D$, and $x_{0}=150$ represents that relays close to $D$. As shown in this figure, the SE performance improves when the number of relays is increased. This improvement benefits from the spatial diversity offered by multiple-relay channels. Thus, given a targeted SE, employing more relays can improve the power efficiency of the implanted node. On the other hand, it is shown that the relay location plays a important role for the distributed relay IBANs. With a given $P_{s}$, the SE performance of the proposed distributed beamforming scheme is sensitive to the relay location. Based on these observation, it is concluded that the relay location is an influential parameter in UWB based IBANs. Choosing relays with proper locations can enhance the system performance effectively.

To further investigate the impact of relay location, the average SE versus $x_{0}$ with different $P_{s}$ in a 2-relay UWB based IBANs is illustrated in Fig. 4. The coordinates of two relays are set to be $\left\{\left(x_{0}, 20\right)\right\}$ and $\left\{\left(x_{0},-20\right)\right\}$, respectively. From this figure, we notice that the optimal $x_{0}$ exists with a fixed $P_{s}$ and the optimal $x_{0}$ varies with $P_{s}$. When the battery power of $S$ is very limited and $P_{S}$ is low, employing the relays close to $P$ can achieve the utmost SE. As $P_{s}$ becomes larger, the optimal $x_{0}$ increases and choosing the relays located near 


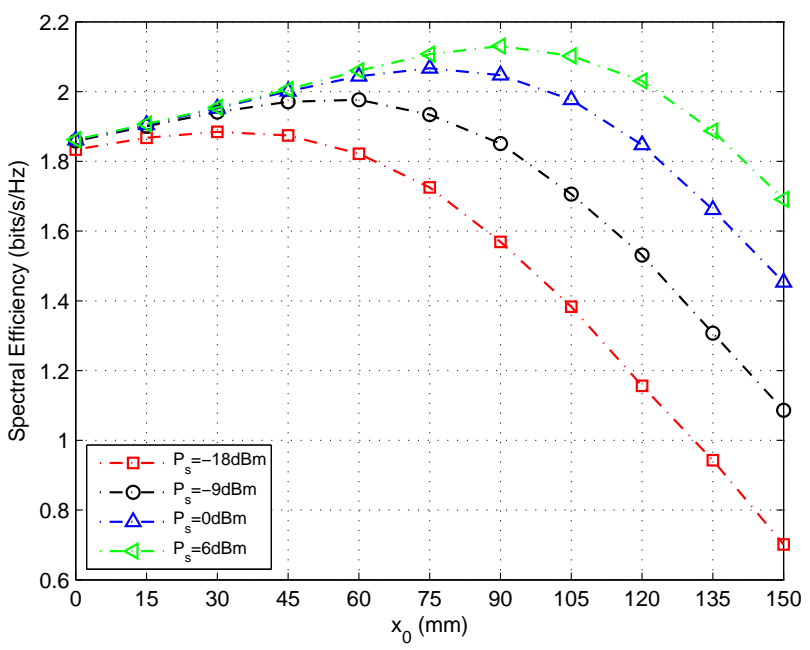

Fig. 4. Average SE versus $x_{0}$ with different $P_{s}$ in a 2-relay network.

the middle of $P$ and $D$ is the best option.

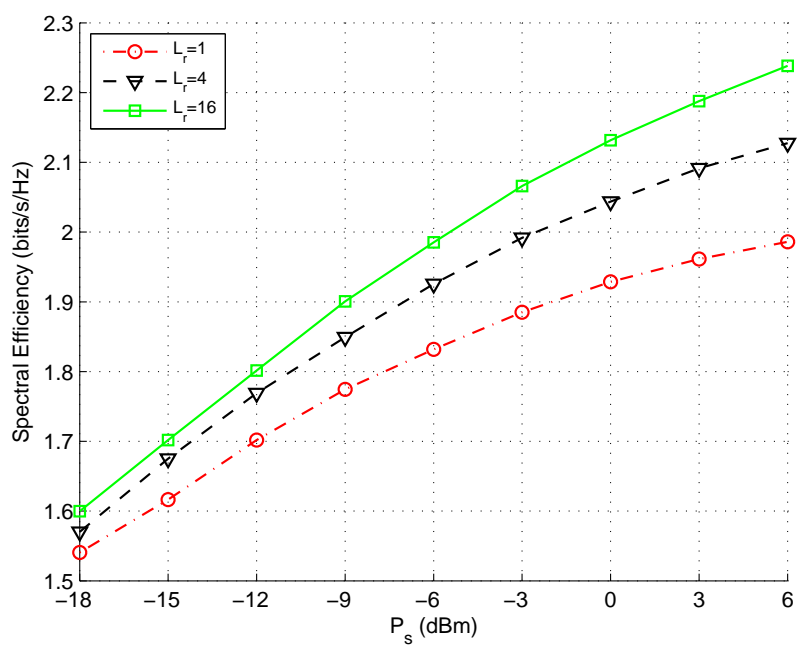

Fig. 5. Average SE versus $P_{s}$ with different $L_{r}$ in a 2-relay network.

Fig. 5 presents the average $\mathrm{SE}$ versus $P_{s}$ with different $L_{r}$ in a 2-relay network, where the two relay locations are set to be same as that in Fig. 2. Obviously, employing more Rake fingers can achieve better SE performance because more multipath gain can be captured. From the power consumption viewpoint, the lifetime of the implanted node can be prolonged at the cost of deploying more Rake fingers at $D$.

In conclusion, the proposed distributed beamforming scheme can be applied to UWB based IBANs effectively, which is superior to other transmission schemes. In distributed relay IBANs, the lifetime of the implanted node can be prolonged at the cost of deploying more relays and more Rake fingers at $D$ with a targeted SE. Moreover, the relay location has an significant impact on the system performance and with different battery power limit of the implanted node, choosing relays with proper locations is an important issue for system performance.

\section{Conclusions}

In this paper, we investigate the SE optimization problem with distributed beamforming in UWB based IBANs. The proposed network beamforming scheme is equivalent to solve a distributed power allocation problem, where each relay properly adjust its own power such that the system SE can be maximized. Numerical examples demonstrate the effectiveness of the proposed distributed beamforming scheme applied to UWB based IBANs. We further show that the relay location is an influential parameter in UWB based IBANs. The system performance with different relay location may have a notable difference. Thus, the effect of relay location cannot be ignored. Moreover, from the power efficiency point of view, it is indicated that the proposed beamforming scheme is an promising way to enhance the power efficiency of the implanted node and prolong its lifetime.

\section{REFERENCES}

[1] IEEE Standard for local and metropolitan area networks-Part 15.6: Body Area Networks, 2012.

[2] M. Patel and J. Wang, "Applications, challenges, and prospective in emerging body area networking technologies," IEEE Wireless Communications, vol. 17 , no. 1,2010 , pp. 80-88.

[3] A. Nosratinia, T. Hunter and A. Hedayat, "Cooperative communication in wireless networks," IEEE Communications Magzine, vol. 42, 2004, pp. 68-73.

[4] Y. Zhao, R. Adve, and T. J. Lim, "Improving amplify-and-forward relay networks: optimal power allocation versus selection," IEEE Transactions on Wireless Communications, vol. 6, Aug. 2007, pp. 3114-3122.

[5] Y. Jing and H. Jafarkhani, "Network beamforming using relays with perfect channel information," IEEE Transactions on information theory, vol. 55, no. 6, June 29, pp. 2499-2517.

[6] V. Havary-Nassab, S. Shahbazpanahi, A. Grami, and Z.-Q. Luo, "Distributed beamforming for relay networks based on second-order statistics of the channel state information," IEEE Transactions on Signal Processing, vol. 56, Sep. 2008, pp. 4306-4316.

[7] H. Chen, A. B. Gershman, and S. Shahbazpanahi, "Filter-and-forward distributed beamforming in relay networks with frequency selective fading," IEEE Transactions on Signal Processing, vol. 58, no. 3, March 2010, pp. 1251-1261.

[8] S. Zhu and K. K. Leung, "Cooperative orthogonal MIMO-relaying for UWB ad-hoc networks," Proc. IEEE Globecom, 2007, pp.5175-5179.

[9] K. Maichalernnukul, T. Kaiser, and F. Zheng "Performance investigation of a UWB relay system using multiple relays with multiple antennas in IEEE 802.15.3a channel," Proc. IEEE VTC, 2009, pp. 1-6.

[10] S. Zhu, K. K. Leung, and A. G. Constantinides, "Distributed cooperative data relaying for diversity in impulse-based UWB ad-hoc networks," IEEE Transactions on Wireless Communications, vol. 8, no. 8, Aug. 2009, pp. 4037-4047.

[11] M. Mondelli, Q. Zhou, X. Ma, and V. Lottici, "A cooperative approach for amplify-and-forward differential transmitted reference IR-UWB relay systems," Proc. IEEE ICASSP, 2012, pp. 2905-2908.

[12] K. Y. Yazdandoost and K. Sayrafian-Pour, "Channel model for body area network (ban)," IEEE P802.15 Working Group for Wireless Personal Area Networks (WPANs), Tech. Rep. Document IEEE802.15-08-078005-0006, February 2009.

[13] A. Khaleghi, R. Chvez-Santiago, and I. Balasingham, "Ultra-wideband statistical propagation channel model for implant sensors in the human chest," IET Microwaves, Antennas and Propagation, vol. 5, no. 15, Dec. 2011, pp. 1805-1812.

[14] L. Yang and G. B. Giannakis, "Analog space-time coding for multiantenna ultra-wideband transmissions," IEEE Trans. Commun, vol. 52, 2004, pp. 507-517.

[15] S. Boyd and L. Vandenberghe, Convex Optimization. Cambridge University Press, 2004. 